\title{
Biochemical networking contributes more to genetic buffering in human and mouse metabolic pathways than does gene duplication
}

T Kitami \& J H Nadeau

Nature Genet. 32, 191-194 (2002).

Published online 5 August 2002; doi:10.1038/ng945

To the authors' dismay, a software bug adversely affected some but not all of the main conclusions of this article. They reported that redundant biochemical networks provide greater genetic buffering than do gene families, on the basis of the rate of gene evolution for genes in the glycolysis/gluconeogenesis and the folate/homocysteine pathways. A bug in software that converted ClustalW alignments into Mega2.1 format considerably affected the data set for these two pathways, which contributed to the second mode of the Ka/Ks distribution (faster evolving genes). This bug did not affect results for other pathways, because those data were analyzed with a different and, unbeknownst to the authors, corrected version of the software. Reanalysis of the entire data set with the corrected software shows that redundant networks do not contribute more to genetic buffering than do gene families. One of the main conclusions from the data should now be that redundant networks provide no greater genetic buffering than do gene families. The fifth line of the abstract should now read "We found that genes with redundant networks evolve at similar rates as did genes without redundant networks." In addition, the glycolysis and gluconeogenesis pathways do not show distinct patterns of variation (average Ka/Ks $=0.108$ ) compared with other pathways. The authors regret this error.

\section{Frequent somatic mutations in PTEN and TP53 are mutually exclusive in the stroma of breast carcinomas}

K Kurose, K Gilley, S Matsumoto, P H Watson, X-P Zhou \& C Eng

Nature Genet. 32, 355-357 (2002).

Published online 15 October 2002; doi:10.1038/ng1013

On the first page, in the third column, the sentence "In two of the nine samples in which we found somatic mutations in TP53 in both the epithelium and stroma, the mutations were discordant between the two compartments (Table $1 a$, samples 17 and 55 ; Fig. $1 a$ )." should be changed slightly to read "In two of the five samples...".

\section{The $\mathrm{K}-\mathrm{Cl}$ cotransporter $\mathrm{KCC} 3$ is mutant in a severe peripheral neuropathy associated with agenesis of the corpus callosum}

H C Howard, D B Mount, D Rochefort, N Byun, N Dupré, J Lu, X Fan, L Song, J-B Rivière, C Prévost, J Horst, A Simonati, B Lemcke, R Welch, R England, F Q Zhan, A Mercado, W B Siesser, A L George, Jr., M P McDonald, J-P Bouchard, J Mathieu, E Delpire \& G A Rouleau

Nature Genet. 32, 384-392 (2002).

Published online 7 October 2002; corrected 21 October 2002 (details online); doi:10.1038/ng1002

The affiliation of B. Lemcke appeared incorrectly. The correct affiliation for B. Lemcke is Institut für Humangenetik, Universität Münster, Münster, Germany. 